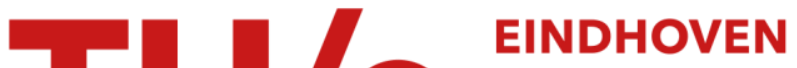 UNIVERSITY OF TECHNOLOGY
}

\section{Deterministic control of radiative processes by shaping the mode field}

Citation for published version (APA):

Pellegrino, D., Pagliano, F., Genco, A., Petruzzella, M., Van Otten, F. W., \& Fiore, A. (2018). Deterministic control of radiative processes by shaping the mode field. Applied Physics Letters, 112(16), [161110]. https://doi.org/10.1063/1.5026803

DOI:

$10.1063 / 1.5026803$

Document status and date:

Published: 16/04/2018

Document Version:

Publisher's PDF, also known as Version of Record (includes final page, issue and volume numbers)

Please check the document version of this publication:

- A submitted manuscript is the version of the article upon submission and before peer-review. There can be important differences between the submitted version and the official published version of record. People interested in the research are advised to contact the author for the final version of the publication, or visit the $\mathrm{DOI}$ to the publisher's website.

- The final author version and the galley proof are versions of the publication after peer review.

- The final published version features the final layout of the paper including the volume, issue and page numbers.

Link to publication

\section{General rights}

Copyright and moral rights for the publications made accessible in the public portal are retained by the authors and/or other copyright owners and it is a condition of accessing publications that users recognise and abide by the legal requirements associated with these rights.

- Users may download and print one copy of any publication from the public portal for the purpose of private study or research.

- You may not further distribute the material or use it for any profit-making activity or commercial gain

- You may freely distribute the URL identifying the publication in the public portal.

If the publication is distributed under the terms of Article $25 \mathrm{fa}$ of the Dutch Copyright Act, indicated by the "Taverne" license above, please follow below link for the End User Agreement:

www.tue.nl/taverne

Take down policy

If you believe that this document breaches copyright please contact us at:

openaccess@tue.nl

providing details and we will investigate your claim. 


\section{Deterministic control of radiative processes by shaping the mode field}

D. Pellegrino, F. Pagliano, A. Genco, M. Petruzzella, F. W. van Otten, and A. Fiore

Citation: Appl. Phys. Lett. 112, 161110 (2018); doi: 10.1063/1.5026803

View online: https://doi.org/10.1063/1.5026803

View Table of Contents: http://aip.scitation.org/toc/apl/112/16

Published by the American Institute of Physics

\section{Articles you may be interested in}

Giant enhancement in Goos-Hänchen shift at the singular phase of a nanophotonic cavity

Applied Physics Letters 112, 161109 (2018); 10.1063/1.5027133

Mid-infrared GaSb-based resonant tunneling diode photodetectors for gas sensing applications Applied Physics Letters 112, 161107 (2018); 10.1063/1.5025531

Boxcar detection for high-frequency modulation in stimulated Raman scattering microscopy

Applied Physics Letters 112, 161101 (2018); 10.1063/1.5022266

Amplification of wavelength-shifting soliton in active photonic crystal fibers

Applied Physics Letters 112, 161105 (2018); 10.1063/1.5009368

Ultrasound liquid crystal lens

Applied Physics Letters 112, 161104 (2018); 10.1063/1.5027131

Abnormal staircase-like I-V curve in InGaN quantum well solar cells

Applied Physics Letters 112, 161102 (2018); 10.1063/1.5018481

\section{PHYSICS TODAY}

MANAGER'S GUIDE

WHITEPAPERS

\section{READ NOW}

PRESENTED BY

Accelerate R\&D with

Multiphysics Simulation 


\title{
Deterministic control of radiative processes by shaping the mode field
}

\author{
D. Pellegrino, ${ }^{1, a)}$ F. Pagliano, ${ }^{1,2}$ A. Genco, ${ }^{1}$ M. Petruzzella, ${ }^{1,2}$ F. W. van Otten, ${ }^{1}$ and A. Fiore ${ }^{1}$ \\ ${ }^{1}$ Department of Applied Physics and Institute for Photonic Integration, Eindhoven University of Technology, \\ Postbus 513, Eindhoven $5600 \mathrm{MB}$, The Netherlands \\ ${ }^{2}$ nanoPHAB, Groene Loper 19, Postbus 513, Eindhoven 5612 AP, The Netherlands
}

(Received 23 February 2018; accepted 9 April 2018; published online 20 April 2018)

\begin{abstract}
Quantum dots (QDs) interacting with confined light fields in photonic crystal cavities represent a scalable light source for the generation of single photons and laser radiation in the solid-state platform. The complete control of light-matter interaction in these sources is needed to fully exploit their potential, but it has been challenging due to the small length scales involved. In this work, we experimentally demonstrate the control of the radiative interaction between InAs QDs and one mode of three coupled nanocavities. By non-locally moulding the mode field experienced by the QDs inside one of the cavities, we are able to deterministically tune, and even inhibit, the spontaneous emission into the mode. The presented method will enable the real-time switching of Rabi oscillations, the shaping of the temporal waveform of single photons, and the implementation of unexplored nanolaser modulation schemes. Published by AIP Publishing. https://doi.org/10.1063/1.5026803
\end{abstract}

Quantum emitters (QEs) interacting with confined electromagnetic fields inside optical cavities represent a versatile system able to provide single-photons on-demand, which are crucial resources in photonic quantum simulators, ${ }^{1}$ optical quantum computers, ${ }^{2}$ and quantum networks. ${ }^{3}$ Solid-state versions of the system-e.g., quantum dots (QDs) in photonic crystal cavities (PCCs) - ensure a good degree of scalability as they are easily integrated with photonic structures with complex functionality. ${ }^{4}$ Indeed, spontaneously emitted single-photons can be efficiently extracted from a nanocavity mode interacting with one exciton, ${ }^{5,6}$ while integrated beamsplitters and phase shifters can provide the photon routing inside the chip. ${ }^{7}$ The small size and high radiative rates achievable in PCCs, while potentially advantageous, also pose practical challenges in their application. In particular, achieving a full control of radiative processes, as realized in microwave cavity quantum electrodynamics (c-QED) systems, ${ }^{8}$ is difficult in PCCs due to their sub-micrometer size. Previous approaches to the dynamic control of c-QED, including the modulation of the cavity-emitter detuning ${ }^{9,10}$ and the tuning of the mode properties, ${ }^{11}$ were also associated with a phase modulation of the produced photons (chirp), which has negative effects on the application in quantum networks ${ }^{12}$ and classical communication systems. ${ }^{13}$

The parameter that quantifies the interaction between a $\mathrm{QE}$ and a target cavity mode within the dipole approximation is the emitter-photon coupling rate $g$, defined as ${ }^{14}$

$$
g=\frac{\overrightarrow{\mathrm{d}} \cdot \overrightarrow{\mathrm{E}}\left(\overrightarrow{\mathrm{r}}_{e m}\right)}{\hbar},
$$

where $\vec{d}$ represents the dipole moment of the transition and $\vec{E}\left(\vec{r}_{e m}\right)$ is the electric field per photon that the emitter experiences at its position $\vec{r}_{e m}$. As $g$ explicitly appears in the expressions for the period of Rabi oscillations and the spontaneous emission (SE) rate, it represents the fundamental parameter underlying all emission processes. However, in

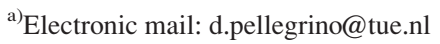

single nanocavities, the rate $g$ is challenging to control since it depends on static quantities as in Eq. (1).

Coupled optical modes (or supermodes ${ }^{15-23}$ ) originated by the interaction of multiple cavities offer a way to nonlocally control the field amplitude $E(\vec{r})$ in one resonator by acting on the others, effectively changing the rate $g$ via Eq. (1). The proof of principle of this approach has been reported in Ref. 11, where the ultrafast control of the SE rate has been achieved in a two-cavity system. However, a limited $g$ tunability (up to a factor $\sqrt{2}$ ) and the aforementioned chirp limit the field modulation when using two cavities. ${ }^{11}$ An approach to overcome these limitations, recently proposed in Ref. 20, relies on an array of three coupled cavities, where an antisymmetric detuning of the outer cavities produces a large change in the field of the supermode inside the third (target) resonator without changing its frequency. ${ }^{21-23}$ Such a system allows the control of the single-photon temporal waveform and the Rabi oscillations in real time, with a modulation speed that solely depends on the detuning technique. ${ }^{20}$

Here, we experimentally implement this concept and demonstrate the full control of the SE of an ensemble of semiconductor quantum dots (QDs) integrated within three coupled cavities. By exploiting the supermodes of the system, we are able to deterministically tailor the mode field experienced by the emitters inside a target cavity, modulating the SE rate from the uncoupled limit to full inhibition.

The system is sketched in Fig. 1(a). A QE represented as a two-level system is weakly coupled to the mode of the target cavity (frequency $\nu_{t}$, loss rate $\kappa_{t}$ ) at a rate $g_{t}$, and spontaneously emits photons into this mode at a rate $\Gamma_{t}=4 g_{t}{ }^{2} / \kappa_{t}^{24}$ when the cavity is isolated. Two tuneable cavities (loss rates $\left.\kappa_{l}, \kappa_{r}\right)$ are then side-coupled to the target cavity at a rate $\eta$, which is large enough so that the cavities are strongly coupled. Here, we specifically assume that $\eta>\kappa_{l}, \kappa_{r}, \kappa_{t}, \gamma_{e m}$ (where $\gamma_{e m}$ is the emitter's spectral linewidth) so that the supermodes are spectrally well separated and the emission into a single mode can be identified. The resonance frequencies of the lateral cavities are blue-shifted and red-shifted 
(a)

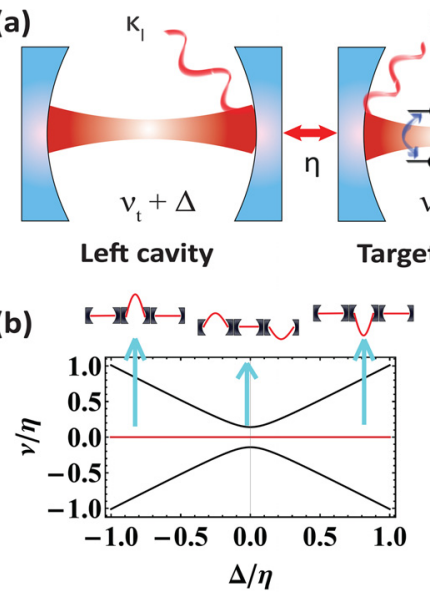

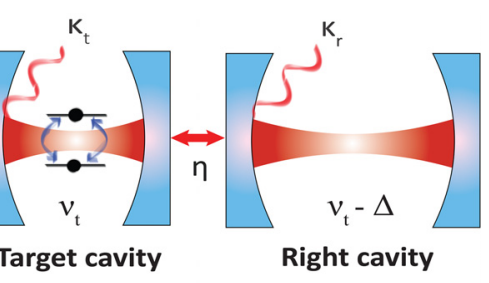

(c)

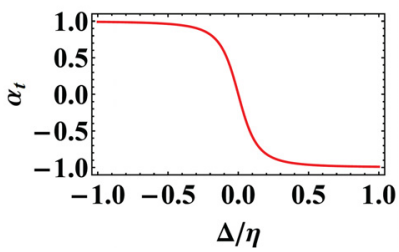

FIG. 1. (a) Sketch of the system. (b) Numerical calculation of the three supemode frequencies vs. the normalized detuning $\Delta / \eta$. The supermode of interest is the central one (red). The field distribution of this supermode is sketched in the top part. (c) $\alpha_{t}$ vs. $\Delta / \eta$. [Parameters used: $\eta=0.1 \mathrm{THz}, \nu_{t}=\kappa_{l}=\kappa_{r}=\kappa_{t}$ $=0 \mathrm{THz}$.]

with respect to $\nu_{t}$ by a quantity $\Delta$, which represents the controllable parameter of the system.

According to the coupled mode theory (CMT) ${ }^{25}$ three supermodes emerge from such an interaction [Fig. 1(b)]. For large detuning $(|\Delta| / \eta \gg 1)$, the cavities are decoupled and the field of the central supermode is localized in the target cavity [Fig. 1(b)-top]. As the detuning $\Delta$ decreases, the resonators couple and the electromagnetic field of this central supermode tends to delocalize in the lateral cavities. At zero detuning, the strong cavity-cavity interaction results in a three-mode anticrossing that leaves the central supermode wavelength [Fig. 1(b), red curve] unaltered. The QEs coupled with this mode experience a detuning-dependent field amplitude $E(\Delta)$ that can be expressed in terms of the uncoupled-cavity modes of the target, left, and right cavities $E_{t}\left(\vec{r}_{e m}\right), E_{l}\left(\vec{r}_{e m}\right), E_{r}\left(\vec{r}_{e m}\right)$ as $E(\Delta)=\alpha_{l}(\Delta) E_{l}\left(\vec{r}_{e m}\right)$ $+\alpha_{t}(\Delta) E_{t}\left(\vec{r}_{e m}\right)+\alpha_{r}(\Delta) E_{r}\left(\vec{r}_{e m}\right)$, where $\alpha_{r, l, t}(\Delta)$ quantify the weight of the original modes in the central supermode. For QEs in the target cavity, $E_{l}\left(\vec{r}_{e m}\right)=E_{r}\left(\vec{r}_{e m}\right)=0$, and therefore $\alpha_{t}(\Delta)$ determines the emitter-photon coupling rate in the target cavity via $g(\Delta)=\alpha_{t}(\Delta) \cdot g_{t}{ }^{20}$ The SE rate of the QE into the coupled mode becomes $\Gamma(\Delta)=4 g^{2}(\Delta) / \kappa(\Delta)$, where $\kappa(\Delta)$ represents the loss rate of the considered supermode, given by $\kappa(\Delta)=\kappa_{l} \cdot\left|\alpha_{l}(\Delta)\right|^{2}+\kappa_{t} \cdot\left|\alpha_{t}(\Delta)\right|^{2}+\kappa_{r} \cdot\left|\alpha_{r}(\Delta)\right|^{2} .{ }^{20}$

In the uncoupled limit [Fig. 1(c), $|\Delta| / \eta \gg 1],\left|\alpha_{t}(\Delta)\right|$ $=1$, and the SE rate is $\Gamma(\Delta)=\Gamma_{t}$. For small detunings, $\left|\alpha_{t}(\Delta)\right|$ decreases until zero at $\Delta=0$, making the central supermode effectively dark. Therefore, the absolute value of $g(\Delta)$, as well as $\Gamma(\Delta)$, can assume every value from the uncoupled case to be zero in a controllable way, with the only experimental requirement of tuneable resonators.

The scheme discussed has a general nature and it is applicable to several experimental implementations, ranging from superconducting circuits to novel opto-mechanical schemes. ${ }^{24}$ In this work, the $g$-tuning is achieved by using PCCs and an ensemble of self-assembled QDs as QEs. The resonances in PCCs can be controlled by several methods including the thermo-optic effect, ${ }^{26}$ the injection of free carriers $^{27}$ or the all-optical Kerr effect. ${ }^{28}$ In this work, we

exploit the thermo-optic effect, which is based on the temperature dependence of the GaAs refractive index and therefore allows the local detuning of the cavity resonances via heating induced by laser irradiation.

Figure 2(a) shows the photonic structure, which consists of an L3 cavity (three missing holes in a hexagonal lattice) evanescently coupled to other two longer L100 resonators. The positions and radii of two holes along the L3 cavity are modified to reduce the loss rate [Fig. 2(a)-bottom]. ${ }^{29}$ Two laser beams [blue spots in Fig. 2(a)-top] with power $P_{l}$ and $P_{r}$, respectively, provide the thermal tuning of the resonances. These spots cause an excitation of the QDs in the lateral cavities, but due to their distant position $(\approx 30 \mu \mathrm{m})$ from the excitation spot, the resulting photon emission is not directly collected. Additionally, air trenches are included in the system to thermally isolate the target cavity from the other ones, and to avoid excessive diffusion of the produced heat which would reduce the effective tuning range. The coupling discussed in this work involves the fundamental mode of the L3 cavity and two Fabry-Perot-like L100 modes, whose frequencies are sufficiently far away from the band edge to avoid localization, which may occur due to the combination of disorder and reduced group velocity. ${ }^{30}$

Figure 2(b) shows the normalized in-plane electric field profile $\left|E_{\text {norm }}\right|$ distributed along the cavities at the frequency of the central supermode, obtained via a two-dimensional (2D) finite element method (FEM) simulation. In the uncoupled cavity limit (left), most of the field is localized inside the L3 cavity, while the smaller field amplitude present in the L100 is due to finite detuning. At zero detuning (right), the electric field redistributes in the lateral cavities, causing a suppression of $\left|E_{\text {norm }}\right|$ in the L3. The residual amount of field present in the target cavity at zero detuning is due to the
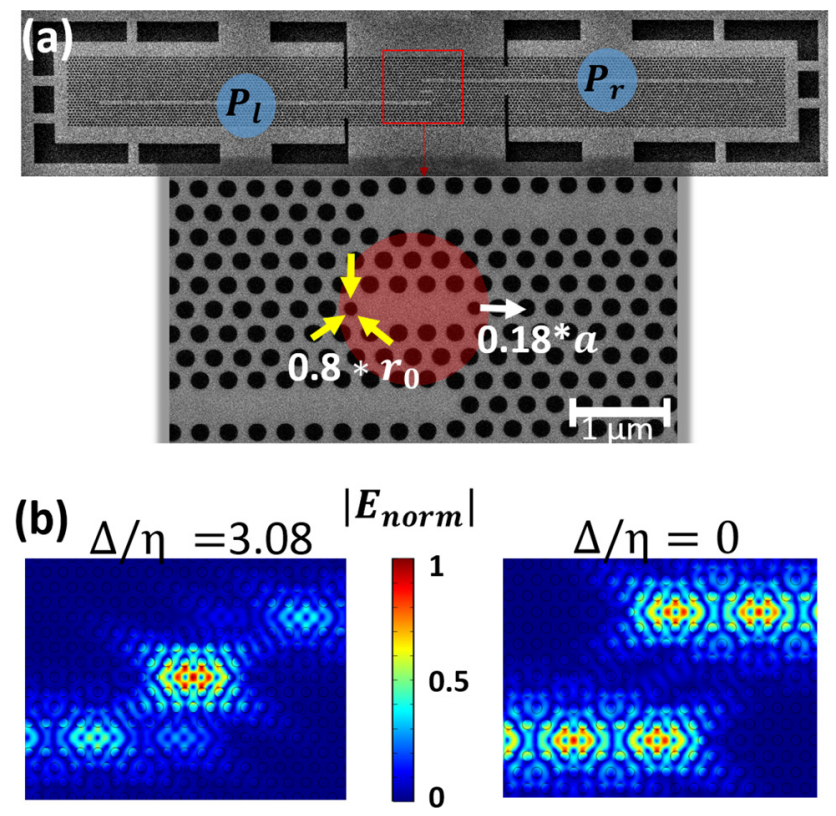

FIG. 2. (a) The photonic structure (top). Two laser spots with power $P_{l}$ and $P_{r}$ (diameter $\left.\approx 8 \mu \mathrm{m}\right)$ provide the heating of the GaAs membrane. Air trenches are visible around the L100 cavities. Close-up of the L3 cavity (bottom). The positions and radii of the first two holes are modified by the indicated amount. The red spot represents the excitation laser. (b) Simulated normalized electric field at $\Delta / \eta=0$ (right) and $\Delta / \eta=3.08$ (left). [Parameters used: lattice constant $\mathrm{a}=340 \mathrm{~nm}$ and hole radius $r_{0}=0.3 * \mathrm{a}$.] 
evanescent fields of the L100 modes penetrating into the L3 resonator [Fig. 2(b)-right]. At the center of the latter cavity, the residual field is $\approx 2.5 * 10^{-4}$ times the field at large detuning $(\Delta / \eta=3.08)$, and this ratio can be further decreased by engineering the intercavity distance.

The fabricated sample consists of a $220 \mathrm{~nm}$-thick GaAs membrane with one layer of self-assembled QDs (an areal density of $200 \mathrm{dots} / \mu \mathrm{m}^{2}$, ground-state emission at $1200 \mathrm{~nm}$ at $77 \mathrm{~K}$ ) embedded in its center. The fabrication of the device involves a dry etching of the $\mathrm{PhC}$ holes in the GaAs membrane and wet etching of the underlying $1.5 \mu \mathrm{m}$-thick $A l_{0.7} G a_{0.3} A s$ sacrificial layer. ${ }^{31}$

In the first part of the experiment, the light emission spectra from QDs are analysed by means of a confocal micro-photoluminescence setup, which has been modified to allow the presence of the detuning spots $\left(\lambda=640 \mathrm{~nm}, P_{\max }\right.$ $\approx 6 \mathrm{~mW}$ ) on the sample. The tuning range achievable with these spots is approximatively $0.3 \mathrm{THz} / \mathrm{mW}$. The QDs are non-resonantly excited by a different laser $(\lambda=780 \mathrm{~nm}$, spot diameter $\approx 2 \mu \mathrm{m}$ ) through a microscope objective and the resulting photoluminescence (PL) is analysed with a spectrometer (focal length $1 \mathrm{~m}$ ) combined with an InGaAs detector array. All measurements are performed at $77 \mathrm{~K}$ to limit the impact of the QD homogeneous broadening, which reduces the emission into the cavity mode due to poor cavity-emitter spectral overlap. ${ }^{32}$

In the experiment, the powers of the detuning lasers were chosen to bias values $\bar{P}_{l}$ and $\bar{P}_{r}$ in order to initially set the L100 resonances at two frequencies, $\nu_{l 0}$ and $\nu_{r 0}$, close to the situation of opposite detuning with respect to the L3 one. In this nearly uncoupled condition, the measured linewidths provide the loss rates of the uncoupled cavities: $\kappa_{l}=0.047 \mathrm{THz}$, $\kappa_{r}=0.053 \mathrm{THz}$, and $\kappa_{t}=0.034 \mathrm{THz}$. Then, for the $n$-th acquired spectra, $P_{l}$ and $P_{r}$ were increased (decreased) by the same amount $\Delta P$ from their respective bias point, so that $P_{l}=\bar{P}_{l}+n \Delta P$ and $P_{r}=\bar{P}_{r}-n \Delta P$.

Figure 3(a) shows a PL color-map obtained by collecting several spectra at different powers $P_{l}$ and $P_{r}$, where $P_{l}$ is shown on the left axis. Seven peaks are presents in the considered spectral region, and we interpret the three central peaks (labelled with 1, 2, and 3) as the supermodes of the coupled L100-L3-L100 system, since they anti-cross at zero detuning as expected. The other four lateral modes are L100 resonances that cross at zero detuning, showing that the L100 cavities couple only via the L3 one. We note that the emission of the detuned L100 cavities is observed due to the finite size of the excitation/collection spot, which partially overlaps with the lateral cavities.

At the anti-crossing point, the peculiar feature of the three-cavity system, the dark mode, is observed as a disappearance of the central PL peak, shown in more detail in Fig. 3(b) (red dots). The same anticrossing behaviour is observed when collecting the PL from one of the lateral cavities, but in this case, as expected, the central supermode does not become dark at resonance [Fig. 3(b), blue dots]. The presence of the dark state in such systems has been observed via transmission measurements in microring-based devices with controllable intercavity coupling, ${ }^{33}$ and via near-field spectroscopy in two-dimensional PCCs. ${ }^{21}$ Our measurement proves that the dependence of the mode field on the detuning
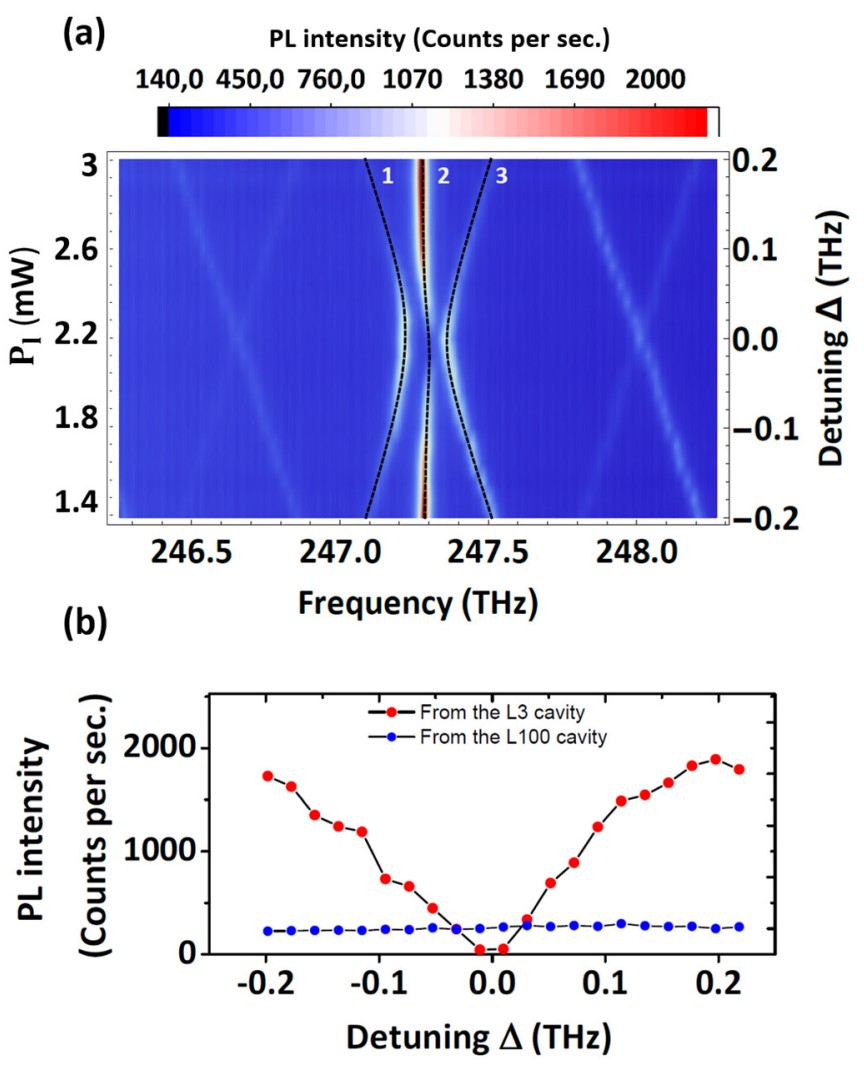

FIG. 3. (a) Experimental PL intensity at different detunings. The black dashed lines represent the calculated frequencies of the supermodes (labelled with 1,2 , and 3 ). The left $y$-axis represents the power $P_{l}$, while the right $y$-axis shows the detuning values extracted from the model. (b) PL intensity at the frequency of the dark mode (supermode 2) when collecting from the target cavity (red dots) and from the left cavity (blue dots).

can be used to tailor the light-matter interaction in the target cavity.

The L100 frequency shifts per power step $\Delta \nu_{l / r} / \Delta P$ of the left/right L100 modes in Fig. 3(a) slightly differ in the absolute value, and this causes a small deviation from the ideal detuning symmetry (in these measurements $\Delta \nu_{l} / \Delta P$ $\left.=-1.03 \cdot \Delta \nu_{r} / \Delta P\right)$. In order to consider the possibility of asymmetric detuning, we define $\Delta$ as $\Delta=\left(\nu_{l}-\nu_{r}\right) / 2$.

As expected, the frequency of the central supermode is nearly unaffected through the entire tuning range. The small dispersive behaviour around the anticrossing point can be explained by small asymmetries in the intercavity coupling due to fabrication imperfection and in the aforementioned difference in the tuning rates. In particular, the experimental coupling rates $\eta_{l}$ and $\eta_{r}$ that characterize the L3-L100 interactions, obtained from two-mode anticrossing measurements when the mode of the other L100 cavity is detuned away from the interaction, are equal to $\eta_{l}=(0.046 \pm 0.001) \mathrm{THz}$ and $\eta_{r}=(0.053 \pm 0.001) \mathrm{THz}$. However, this dispersive behaviour of the central mode, which is potentially detrimental for applications that require dispersion-free resonances, can be compensated by properly changing the values of the detuning slopes $\Delta \nu_{l / r} / \Delta P$.

The black dashed lines in Fig. 3(a) represent the supermode frequencies calculated with CMT analysis (right yaxis), which well reproduce the measured spectra when the experimental asymmetric coupling and detuning rates are taken into account. 
Although the PL data in Fig. 3 clearly shows the suppression of SE in the dark mode, it is not trivial to relate the changes in the PL intensity to the rate $g(\Delta)$. Indeed, the measured PL intensity is not only related to $g(\Delta)$, but also depends on the collection efficiency, which changes with the detuning due to field variations. Therefore, extracting the SE rate from the QD decay rates is required to quantify the changes in $g(\Delta)$.

The decay dynamics of the QDs are measured via a time-correlated single-photon counting (TCSPC) experiment by using a pulsed laser $(\lambda=760 \mathrm{~nm}$, a repetition rate of $80 \mathrm{MHz}$, a pulse width of $70 \mathrm{ps}$, and average power of $1 \mu \mathrm{W}$ ) as excitation and a superconducting single-photon detector $\left(\mathrm{SSPD}^{34}\right)$. The PL signal was filtered at the central supermode frequency by using grating of the spectrometer as a filter (full-width half-maximum $\approx 0.02 \mathrm{THz}$ ).

Figure 4(a) shows a comparison of the relevant decay curves. Away from photonic structures, the QDs are characterized by a radiative decay $\tau_{\text {bulk }}=(0.89 \pm 0.02) \mathrm{ns}$, similar to previously reported values of InAs QDs emitting at $1300 \mathrm{~nm}^{35}$ The emission into the leaky modes of the photonic crystal (black curve), measured away from the cavity, has a decay time $\tau_{\text {leaky }}=(2.45 \pm 0.10)$ ns. The decay time $\tau_{-0.28 \mathrm{THz}}=(0.61 \pm 0.02) \mathrm{ns}$ of the QDs in the target cavity for large detunings (green curve) shows an increase in the photon emission rate that indicates a Purcell-enhanced emission. As the detuning is reduced, the SE rate decreases as expected. The data corresponding to $\Delta=-0.08 \mathrm{THz}$ (blue curve) display a decay time $\tau_{-0.08 \mathrm{GHz}}=(1.14 \pm 0.03) \mathrm{ns}$ that is longer than $\tau_{\text {bulk }}$, which indicates inhibition of lightmatter interaction. Around zero detuning, the reduced PL intensity shown in Fig. 3(a) together with the residual PL
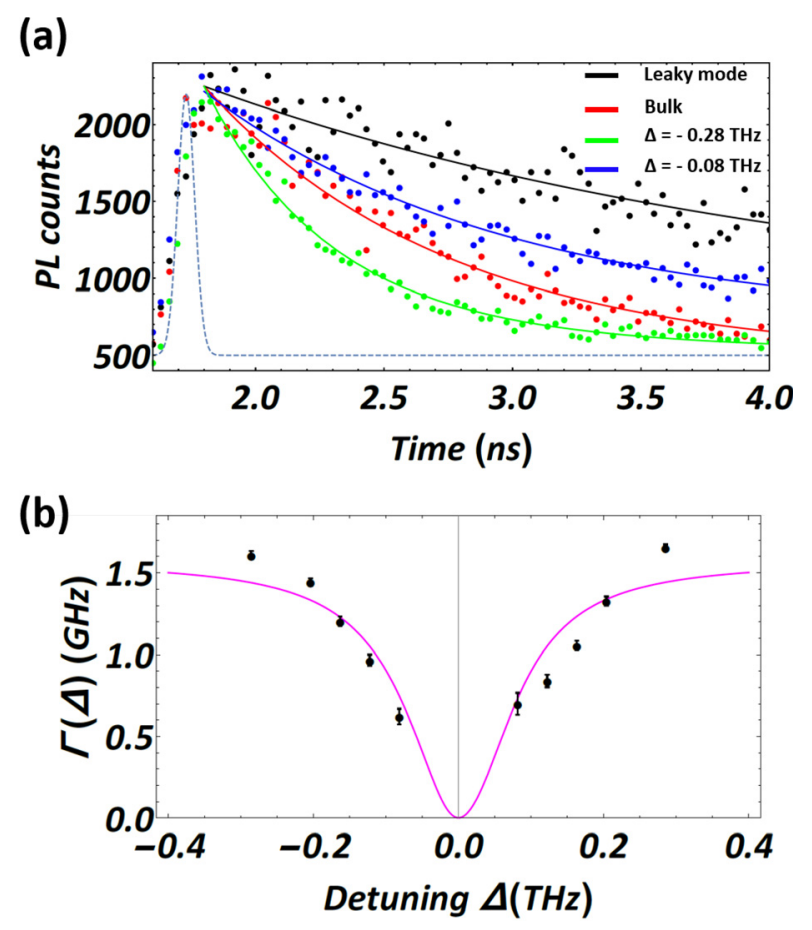

FIG. 4. Decay time measurements. (a) Comparison among the relevant exponential decays. The dashed line represents the instrument response function (full width half maximum $76 \mathrm{ps}$ ), obtained with a time-resolved measurement of the excitation laser pulse. (b) SE rates $\Gamma(\Delta)$ (dots) and the fitted curve (magenta). collected from the lateral cavities, makes the measurement of the QD emission rate into the central supermode impossible. Indeed, the PL collected from the lateral cavities becomes dominant with respect to the reduced PL intensity produced by the QDs inside the L3 resonator around $\Delta=0$. To confirm that the observed change in radiative lifetime is related to field tuning, several curves are measured at different detunings, and the SE rates in the cavity mode are derived from the decay times $\tau_{\Delta}$ as $\Gamma(\Delta)=1 / \tau_{\Delta}-1 / \tau_{\text {leaky }}$. The obtained values [Fig. 4(b)] are fitted with the relation $\Gamma(\Delta)=4 g^{2}(\Delta) / \kappa(\Delta)$. Here, we set $g(\Delta)=g_{t} \cdot \alpha_{t}(\Delta)$ and calculate the $\alpha_{t}(\Delta)$ and $\kappa(\Delta)$ values with the CMT model with the experimental loss, coupling, and detuning rates obtained from the spectra in Fig. 3(a), so that $g_{t}$ represents the only free parameter. The good agreement between the data and the fit in Fig. 4(b) indicates that the emitter-cavity interaction rate can be tuned between the uncoupled cavity limit, $g_{t}=3.65 \mathrm{GHz}$, and a minimum value close to zero. The interaction rate at resonance can only be due to the residual field at zero detuning (which produces a coupling estimated as $\approx 2.5 * 10^{-4} g_{t}$ ), and to imperfect experimental control of the detuning. From the calculated $\alpha_{t}(\Delta)$ dependence, we estimate that a control of the detuning within $\Delta_{\max }=0.001$ $\mathrm{THz}$ is for example needed to keep $g<10^{-2} g_{t}$. The detuning can be monitored by fitting the PL peaks from the lateral cavities and its control is ultimately limited by the signal-tonoise of this measurement and the stability of the pump laser.

In conclusion, we demonstrated a mode field modulation in a three-cavity system that allows complete control of the light-matter interaction inside the target resonator. Spectral- and temporal-resolved analysis of the PL showed controllable tuning of the emitter-photon coupling rate $g$, while the dispersion-free nature of the dark mode prevents frequency variations of the emitted photons. Combined with ultrafast tuning techniques, ${ }^{27,28}$ these features open the way to the control of Rabi oscillations in real time and to the shaping of the temporal waveform of single photons in the $\approx 100$ ps timescale. An additional exciting perspective is the control of the cavity-emitter dynamics around the exceptional point. ${ }^{36}$

This work is part of the research programme of the Foundation for Fundamental Research on Matter (FOM), which is part of the Netherlands Organisation for Scientific Research (NWO). D.P. and A.F. acknowledge E. Verhagen and M. Cotrufo for the useful discussions and S. Birindelli for the help in setting up the experiment. A.G. acknowledges funding from the University of Salento (Dipartimento di Matematica e Fisica "Ennio De Giorgi," Lecce, Italy) and the Erasmus+ programme of the European Union.

\footnotetext{
${ }^{1}$ A. Aspuru-Guzik and P. Walther, Nat. Phys. 8, 285 (2012).

${ }^{2}$ E. Knill, R. Laflamme, and G. J. Milburn, Nature 409, 46 (2001).

${ }^{3}$ J. I. Cirac, P. Zoller, H. J. Kimble, and H. Mabuchi, Phys. Rev. Lett. 78, 3221 (1997).

${ }^{4}$ C. P. Dietrich, A. Fiore, M. G. Thompson, M. Kamp, and S. Hofling, Laser Photonics Rev. 10, 870 (2016).

${ }^{5}$ P. Lodahl, S. Mahmoodian, and S. Stobbe, Rev. Mod. Phys. 87, 347 (2015).

${ }^{6}$ S. Buckley, K. Rivoire, and J. Vuckovic, Rep. Prog. Phys. 75, 126503 (2012).
} 
${ }^{7}$ L. Midolo, S. L. Hansen, W. Zhang, C. Papon, R. Schott, A. Ludwig, A. D. Wieck, P. Lodahl, and S. Stobbe, Opt. Express 25, 33514 (2017).

${ }^{8}$ Y. Yin, Y. Chen, D. Sank, P. J. J. O’Malley, T. C. White, R. Barends, J. Kelly, E. Lucero, M. Mariantoni, A. Megrant, C. Neill, A. Vainsencher, J. Wenner, A. N. Korotkov, A. N. Cleland, and J. M. Martinis, Phys. Rev. Lett. 110, 107001 (2013).

${ }^{9}$ F. Pagliano, Y. Cho, T. Xia, F. van Otten, R. Johne, and A. Fiore, Nat. Commun. 5, 5786 (2014).

${ }^{10}$ R. Bose, T. Cai, K. R. Choudhury, G. S. Solomon, and E. Waks, Nat. Photonics 8, 858 (2014).

${ }^{11}$ C.-Y. Jin, R. Johne, M. Y. Swinkels, T. B. Hoang, L. Midolo, P. J. van Veldhoven, and A. Fiore, Nat. Nanotechnol. 9, 886 (2014).

${ }^{12}$ R. Johne and A. Fiore, Phys. Rev. A 84, 053850 (2011).

${ }^{13}$ G. P. Agrawal, Fiber-Optic Communication Systems (John Wiley \& Sons Inc., 2002).

${ }^{14}$ C. Gerry and P. Knight, Introductory Quantum Optics (Cambridge University Press, Cambridge, 2004).

${ }^{15}$ S. Hughes, Phys. Rev. Lett. 98, 083603 (2007).

${ }^{16}$ A. Majumdar, A. Rundquist, M. Bajcsy, and J. Vuckovic, Phys. Rev. B 86, 045315 (2012).

${ }^{17}$ J. Scheuer, G. T. Paloczi, J. K. S. Poon, and A. Yariv, Opt. Photonics News 16, 36 (2005).

${ }^{18}$ A. R. A. Chalcraft, S. Lam, B. D. Jones, D. Szymanski, R. Oulton, A. C. T. Thijssen, M. S. Skolnick, D. M. Whittaker, T. F. Krauss, and A. M. Fox, Opt. Express 19, 5670 (2011).

${ }^{19}$ C. Yang, Y. Hu, X. Jiang, and M. Xiao, Phys. Rev. A 95, 033847 (2017).

${ }^{20}$ R. Johne, R. Schutjens, S. Fattah poor, C.-Y. Jin, and A. Fiore, Phys. Rev. A 91, 063807 (2015).

${ }^{21}$ N. Caselli, F. Riboli, F. La China, A. Gerardino, L. Li, E. H. Linfield, F. Pagliano, A. Fiore, F. Intonti, and M. Gurioli, ACS Photonics 2, 565 (2015).
${ }^{22}$ M. Cotrufo, E. Verhagen, and A. Fiore, Proc. SPIE 10111, 1011128 (2017).

${ }^{23}$ M. Cotrufo, A. Fiore, and E. Verhagen, Phys. Rev. Lett. 118, 133603 (2017).

${ }^{24}$ L. C. Andreani, G. Panzarini, and J.-M. Gérard, Phys. Rev. B 60, 13276 (1999).

${ }^{25}$ H. A. Haus and W. Huang, Proc. IEEE 79(10), 1505-1518 (1991).

${ }^{26}$ H. M. H. Chong and R. M. De La Rue, IEEE Photonics Technol. Lett. 16, 1528 (2004).

${ }^{27}$ I. Fushman, E. Waks, D. Englund, N. Stoltz, P. Petroff, and J. Vučković, Appl. Phys. Lett. 90, 091118 (2007).

${ }^{28}$ V. Eckhouse, I. Cestier, G. Eisenstein, S. Combrie, G. Lehoucq, and A. De Rossi, Opt. Express 20, 8524 (2012).

${ }^{29}$ Y. Akahane, T. Asano, B.-S. Song, and S. Noda, Nature 425, 944 (2003).

${ }^{30}$ S. Hughes, L. Ramunno, J. F. Young, and J. E. Sipe, Phys. Rev. Lett. 94, 033903 (2005).

${ }^{31}$ M. Francardi, L. Balet, A. Gerardino, C. Monat, C. Zinoni, L. H. Li, B. Alloing, N. Le Thomas, R. Houdré, and A. Fiore, Phys. Status Solidi C 3, 3693 (2006).

${ }^{32}$ A. Auffèves, J.-M. Gérard, and J.-P. Poizat, Phys. Rev. A 79, 053838 (2009).

${ }^{33}$ C. Yang, X. Jiang, Q. Hua, S. Hua, Y. Chen, J. Ma, and M. Xiao, Laser Photonics Rev. 11(2), 1600178 (2017).

${ }^{34}$ See http://www.scontel.ru/ for more information about "SSPD Used" (last accessed February 23, 2018).

${ }^{35}$ L. Midolo, F. Pagliano, T. B. Hoang, T. Xia, F. W. M. van Otten, L. H. Li, E. H. Linfield, M. Lermer, S. Höfling, and A. Fiore, Appl. Phys. Lett. 101, 091106 (2012).

${ }^{36}$ R. El-Ganainy, K. G. Makris, M. Khajavikhan, Z. H. Musslimani, S. Rotter, and D. N. Christodoulides, Nat. Phys. 14, 11 (2018). 